\title{
Einander in Achtung begegnen
}

\author{
Ulrich Hemel
}

Nicht Gewinn, sondern Bedarfsdeckung muss das erste Prinzip wirtschaftlichen Handelns sein. Wert und Werte bestimmen auch den Alltag sozialer Organisationen. Deren Führungskräfte müssen zweckrationales und kommunikatives Verhalten immer wieder neu in das richtige Gleichgewicht bringen.

Die Skandale, die die Republik immer wieder erschüttern, werfen die Frage auf, ob wirtschaftliche Verantwortung und ethisches Handeln nicht von Haus aus Widersprüche sind. Zumindest der jahrelang gültige Grundkonsens über die Grenzen des Erlaubten erscheint gründlich infrage gestellt.

Es wäre andererseits eigentümlich, wenn ein großer Lebensbereich wie die Wirtschaft mit ihren Millionen von Arbeitsplätzen unter einen ethischen Generalverdacht gestellt würde. Nicht zufällig heißt es auf dem II. Vatikanischen Konzil in der Konstitution »Gaudium et Spes« (Nr. 63), der Mensch sei »Urheber, Mittelpunkt und Ziel aller Wirtschaft «. Damit wird in Erinnerung gerufen, dass Wirtschaften der Bedarfsdeckung dient und allein in dieser Hinsicht durchaus mit ethischen Zwecken verbunden werden kann. Auch eine noch so strikte Gewinnorientierung privatwirtschaftlicher Unternehmen kann nicht verdecken, dass nicht Gewinn, sondern Bedarfsdeckung das erste Prinzip wirtschaftlichen Handelns ist.

Gute Unternehmen werden dabei wirtschaftliche und ethische Wertschöpfung miteinander verbinden. Es versteht sich von selbst, dass zweckrational ausgerichtete Unternehmen auf eine optimale Wertschöpfung hin ausgerichtet sind. Darüber hinaus leisten Unternehmen und in Unternehmen handelnde Personen immer dann einen Beitrag zu ethischer Wertschöpfung, wenn sie durch ihr verlässliches Handeln Vertrauen schaffen, erhalten oder mehren - und zwar bei Mitarbeitern und Mitarbeiterinnen ebenso wie bei Kunden, Lieferanten und Eigentümern.

So wie es allgemein gültige ethische Prinzipien gibt, so besteht auch im wirtschaftlichen Bereich ein Vorrang für Werte wie Vertrauen und transparente Kommunikation. Dazu kommen die Forderungen nach Professionalität, optimaler Wertschöpfung und sinnvoller Strategie. Gelebt werden können diese Werte allerdings nur durch das Handeln einzelner Personen, mag auch deren Einbindung in Rollen und Funktionen das entscheidende Moment persönlicher Verantwortung gelegentlich verdunkeln.

Persönliche Verantwortung greift auf allen Ebenen und Hierarchiestufen. Sie gilt auch für Organisationseinheiten im sozialen Bereich. Wer meint, persönliche Verantwor-

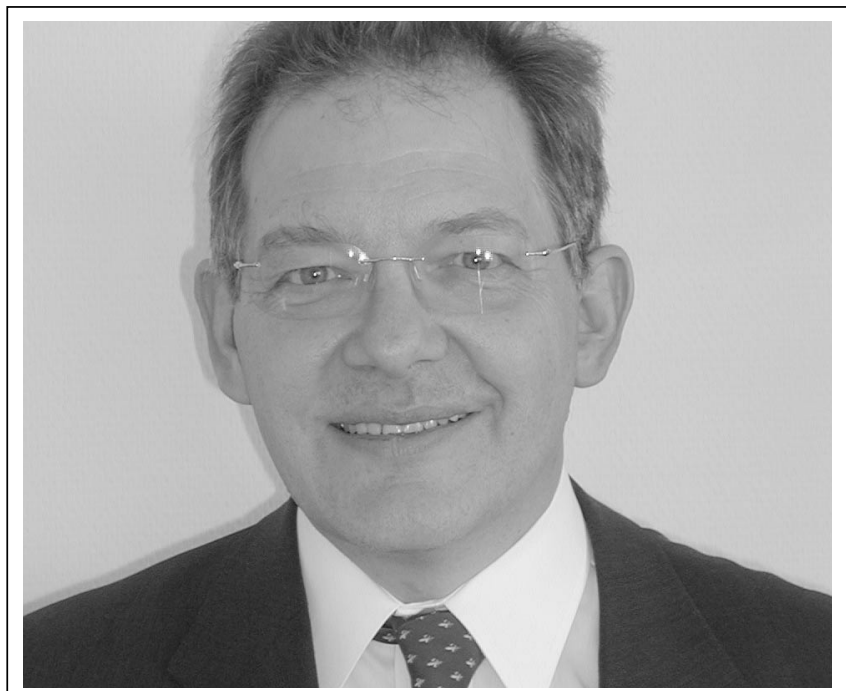

Prof. Dr. Dr. Ulrich Hemel verbindet die Erfahrung aus dem Topmanagement großer Unternehmen mit theologischer und ethischer Reflexion. Nach einer Tätigkeit als Unternehmensberater bei »The Boston Consulting Group « und in der Industrie, u. a. als Vorstandsvorsitzender der Paul Hartmann AG, ist der 49-Jährige heute Vorsitzender der Geschäftsleitung der Süddekor GmbH (2D-Holding), Vorstandsvorsitzender des Forschungsinstituts für Philosophie in Hannover und außerplanmäßiger Professor für Katholische Theologie an der Universität Regensburg. Für sein Buch »Wert und Werte» erhielt er im Oktober 2005 auf der Buchmesse Frankfurt den Preis der Financial Times Deutschland für das »Wirtschaftsbuch des Jahres « in der Kategorie Ethik.

tung delegieren oder hinter angeblichen Sachzwängen verstecken zu können, begibt sich auf das gefährliche Feld der Illusion. Wenn Konflikte auftreten, hängt es immer noch vom Ton ab, der die Musik macht. Und wenn die eigene Ohnmacht größer ist als die verfügbaren Handlungsmittel, wird es unausweichlich sein, sich über die Bedeutung eines Konflikts persönlich klar zu werden. In der Regel ist die persönliche Integrität ein so hoher Wert, dass sogar das Verlassen des Feldes durch Rückzug oder sogar durch die Suche nach einer anderen Arbeitsmöglichkeit geboten sein kann.

Typischerweise folgen Unternehmen ihrer spezifischen Rationalität auf dem von ihnen gewählten Feld. Ein Handelsunternehmen tickt anders als ein Produktionsbetrieb. Ein Gastronomiebetrieb hat andere Gesetze als ein 
Schwimmbad. Gleichwohl gibt es ein verbindendes Element privatwirtschaftlicher Unternehmen: Sie alle folgen dem Zweck des optimalen Wirtschaftens, das auf Gewinn ausgerichtet ist. Sie richten sich daher am Leitbild zweckrationalen Handelns aus. Solches zweckrationale Handeln wirft grundsätzlich und immer wieder die Frage auf, ob eine bestimmte Aktion letztlich dem Unternehmenszweck dient oder nicht, ob sie sich also direkt oder indirekt »lohnt .

Die Frage nach der Menschlichkeit des Handelns kommt unter dieser Perspektive idealtypisch erst in zweiter Folge ins Blickfeld. Da auch im stärksten rational getriebenen Betrieb Menschen mit ihren spezifischen Ängsten, Bedürfnissen und Interessen zusammenwirken müssen, lässt sich die Frage nach ethischen Prinzipien des Handelns dennoch nicht ausklammern. Die spezielle Art und Weise, wie ein Unternehmen nach innen und nach außen agiert, kommt als »Geist « eines Unternehmens zum Ausdruck. Der ethische Lebensstil eines Unternehmens wirkt dann auf den Managementstil seiner Führungskräfte zurück — und umgekehrt. Über Fachkenntnisse hinaus ist daher die kulturelle Passung zwischen Führungskraft und Unternehmen ein wesentliches Kriterium der internen Beförderung oder der Einstellung von außen.

Aus der Blickrichtung der Typologie des Handelns steht einer zweckrationalen Ausrichtung eine eher kommunikative gegenüber. Dabei geht es um Gemeinschaftserfahrung, Kommunikation, Teilhabe und sozialen Austausch ohne direkte Ergebnisorientierung, beispielsweise beim Abendessen mit Freunden oder in der Familie.

Es gibt aber bestimmte Organisationen, die zweckrationales und kommunikatives Verhalten in schwer bestimmbarer Weise mischen. Dazu gehören beispielsweise Schulen und Kirchen, aber auch bestimmte Bildungseinrichtungen und soziale Handlungsfelder. Viele Menschen in solchen Organisationen arbeiten mit einem idealen, vielleicht auch idealistischen Anspruch. Sie machen sich nicht immer klar, welchen Raum kommunikatives und welchen zweckrationales Handeln einnimmt und einnehmen kann. Der auf sozialen Organisationen lastende Kostendruck drängt allerdings in letzter Zeit die scheinbar zwecklosen, aber eben doch Beziehung festigenden kommunikativen Zeiten und Aktivitäten enorm zurück.

Ins Extrem getrieben wäre eine scheinbar ausschließlich zweckrationale Schule eine reine Paukanstalt, ein zweckrationales Kinderheim eine Verwahrstation. Die Beispiele zeigen, wie sehr es der Sensibilität von Führungskräften bedarf, das bestmögliche Gleichgewicht zwischen beiden Handlungstypen in Institutionen zu finden, die auf ein starkes Ineinander von kommunikativen und zweckrationalen Elementen angewiesen sind.

Gefahren liegen dabei sowohl in der idealistischen Selbstausbeutung wie in der ethischen Bevormundung, die immer noch oder schon wieder meint, Entscheidungen über das Wohlbefinden Dritter fällen zu können, ohne die subjektiven Präferenzen der gemeinten Personen einzubeziehen. Umgekehrt wäre es auch fahrlässig, Grenzen einer partizipativen Entscheidungsfindung zu leugnen. Zieht sich eine Führungskraft auf die Rolle eines Konsenssuchers und Moderators paralleler Diskurse zurück, wird sie auch in sozialen Bereichen ihrer Verantwortung als Impulsgeber und Entscheider nicht gerecht. Ethische Verantwortung in der Führung heißt eben auch Verantwortung für eigene Entscheidungen zu übernehmen, und zwar inklusive der Gefahr und Nebenwirkung, dass nicht alle Entscheidungen im Konsens zu treffen sind.

Ethische Professionalität für Führungskräfte in sozialen Institutionen heißt dann vor allem, die Kunst des Zuhörens ebenso zu pflegen wie die Kunst, gelegentlich nein zu sagen und eine Kultur der Konfliktfähigkeit zu fördern. Weder autokratische Entscheidungseinsamkeit noch ewige Diskursorientierung taugen hier als Idealbilder von Führung, sondern vielmehr jene aufmerksame Balance zwischen zweckrationalen und kommunikativen Handlungsformen, die tiefe Sachkenntnis mit hoher Achtung vor der Einzigartigkeit jeder handelnden Person in ihrer spezifischen Lebenswelt verbindet.

Genau darin besteht die persönliche Verantwortung einer ethisch orientierten Führungskraft im sozialen Bereich.

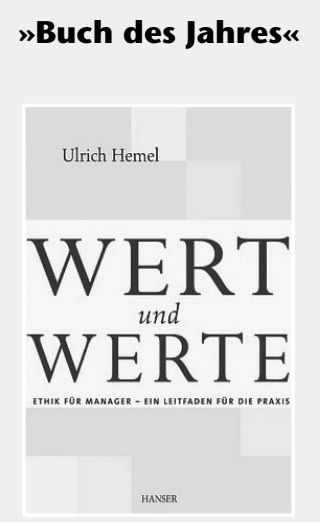

Das Präsidium des Bundesverbandes Deutscher Unternehmensberater BDU e. V. hat dem Manager und Theologen Prof. Dr. Dr. Ulrich Hemel die Auszeichnung »BDU-Buch des Jahres 2005 « für sein Werk »Wert und Werte« verliehen.

Der Autor mache deutlich, dass ohne ethische Grundlagen verantwortungsbewusstes und qualitatives Handeln nicht denkbar

ist. Nur wer diese Spielregeln beachte, könne dauerhaft erfolgreich sein. Wer das Buch gelesen habe, bekomme ein grundlegend anderes Verhältnis zu seinen Entscheidungen, sagte BDU-Präsident Rémi Redley. Hemel sei darüber hinaus nicht nur ein brillanter Analytiker, sondern lebe als Mensch vor, dass sich erfolgreiches und werterorientiertes Handeln vereinbaren lassen. Werte spielten gerade in Zeiten des Umbruchs eine besondere Rolle, betonte der BDU-Präsident. Nur wenn sich die Führungskräfte in Politik und Wirtschaft ihrer Vorbildfunktion wieder bewusst würden und danach handelten, ließe sich ihr angeschlagenes Image in der Öffentlichkeit wieder herstellen.

In seiner Dankesrede sagte Hemel, dass ihm als Manager und Theologen daran gelegen sei, eine Brücke zwischen den beiden Welten zu schlagen, denn sie stellten keinen Widerspruch in sich dar. Ebenso wie der wirtschaftliche Wert ließen sich auch Werte in Form von Vertrauenszuwachs oder Vertrauensschwund messen.

Ulrich Hemel: Wert und Werte. Ethik für Manager ein Leitfaden für die Praxis. Carl Hanser Verlag. München 2005. 306 Seiten. 24,90 Euro.

ISBN 3-446-22813-6. 